\title{
Molecular dynamics simulation of real-fluid mutual diffusion coefficients with the Lennard-Jones potential model
}

\author{
Richard L. Rowley
}

rowley@byu.edu

\section{J. M. Stoker}

Follow this and additional works at: https://scholarsarchive.byu.edu/facpub

Part of the Chemical Engineering Commons

\section{Original Publication Citation}

Stoker, J. M. and R. L. Rowley. "Molecular dynamics simulation of real-fluid mutual diffusion coefficients with the Lennard-Jones potential model." The Journal of Chemical Physics 91 (1989): 367-3676

\section{BYU ScholarsArchive Citation}

Rowley, Richard L. and Stoker, J. M., "Molecular dynamics simulation of real-fluid mutual diffusion coefficients with the Lennard-Jones potential model" (1989). Faculty Publications. 735.

https://scholarsarchive.byu.edu/facpub/735 


\title{
Molecular dynamics simulation of real-fluid mutual diffusion coefficients with the Lennard-Jones potential model
}

\author{
J. M. Stokera) and R. L. Rowley ${ }^{\text {b) }}$ \\ Department of Chemical Engineering, Brigham Young University, Provo, Utah 84602
}

(Received 18 April 1989; accepted 6 June 1989)

\begin{abstract}
Mutual diffusion coefficients for selected alkanes in carbon tetrachloride were calculated using molecular dynamics and Lennard-Jones ( $\mathrm{LJ}$ ) potentials. Use of effective spherical $\mathrm{LJ}$ parameters is desirable when possible for two reasons: (i) computer time is saved due to the simplicity of the model and (ii) the number of parameters in the model is kept to a minimum. Results of this study indicate that mutual diffusivity is particularly sensitive to the molecular size cross parameter, $\sigma_{12}$, and that the commonly used Lorentz-Berthelot rules are inadequate for mixtures in which the component structures differ significantly. Good agreement between simulated and experimental mutual diffusivities is obtained with a combining rule for $\sigma_{12}$ which better represents these asymmetric mixtures using pure component $\mathbf{L} \mathbf{J}$ parameters obtained from self-diffusion coefficient data. The effect of alkane chain length on the mutual diffusion coefficient is correctly predicted. While the effects of alkane branching upon the diffusion coefficient are comparable in size to the uncertainty of these calculations, the qualitative trend due to branching is also correctly predicted by the MD results.
\end{abstract}

\section{INTRODUCTION}

Molecular dynamics (MD) simulation has become a relatively standard method for studying intradiffusion coefficients of real fluids composed of relatively simple molecules. The term intradiffusion, as used in this paper, includes self-diffusion of molecules in pure components and tracer diffusion of individual molecules in equilibrium mixtures. Perhaps somewhat surprising is the paucity of MD studies of mutual or interdiffusion. In fact, the feasibility of calculating accurate mutual diffusion coefficients with MD simulations $^{1-3}$ has only recently been established. This is primarily due to the inherently poorer statistics which result when computing collective properties rather than one-particle properties. The reason for the larger uncertainties in calculated mutual diffusivities can be seen from the equilibrium MD formulation of the diffusion coefficients ${ }^{2-6}$ in terms of either the Green-Kubo (GK) or the mean square displacement (MSD) relations. For intradiffusion coefficients these relationships are

$$
\begin{aligned}
& D_{i}=\frac{1}{3} \int_{t_{v}}^{\infty}\left\langle\mathbf{v}_{i}\left(t_{0}\right) \mathbf{v}_{i}\left(t_{0}+t\right)\right\rangle d t, \\
& D_{i}=\frac{1}{6} \lim _{t \rightarrow \infty} \frac{d}{d t}\left\langle\left[\mathbf{r}_{i}\left(t_{0}\right)-\mathbf{r}_{i}\left(t_{0}+t\right)\right]^{2}\right\rangle,
\end{aligned}
$$

respectively. Here $\mathbf{v}_{i}$ is velocity and $\mathbf{r}_{i}$ is position of particle $i$. The brackets in this case indicate not only a time average, but also a particle average, since each molecule of a particular component may be used to obtain a replicate average of the time correlation function. The corresponding relationships for mutual diffusion coefficients are

$$
D_{i j}=\frac{Q}{3 N c_{i} c_{j}} \int_{t_{i j}}^{\infty}\left\langle J_{i j}\left(t_{0}\right) J_{i j}\left(t_{0}+t\right)\right\rangle d t
$$

\footnotetext{
a) Present address: Vista Chemical Co., Ponca City, Oklahoma.

b) Author to whom all correspondence should be addressed.
}

$$
\begin{aligned}
D_{i j}= & \frac{Q}{6 N c_{i} c_{j}}\left(\frac{m_{i}}{m_{j}} c_{i}+c_{j}\right)^{2} \lim _{t \rightarrow \infty} \frac{d}{d t}\left\langle\left[\sum_{k=1}^{N_{i}} \mathbf{r}_{k}\left(t_{0}\right)\right.\right. \\
& \left.\left.-\sum_{k=1}^{N_{i}} \mathbf{r}_{k}\left(t_{0}+t\right)\right]^{2}\right),
\end{aligned}
$$

where

$$
\begin{aligned}
& J_{i j}(t)=c_{j} \sum_{k=1}^{N_{i}} \mathbf{v}_{k}(t)-c_{i} \sum_{m=1}^{N_{j}} \mathbf{v}_{m}(t), \\
& Q \equiv 1+\left(\frac{\partial \ln \gamma_{1}}{\partial \ln x_{1}}\right)_{T, P},
\end{aligned}
$$

$c_{i}$ is number density of $i, N_{i}$ is number of $i$ particles with $\Sigma N_{i}$ $=N, \gamma_{1}$ is the activity coefficient of component 1 and $x_{1}$ is the mole fraction of component 1 . Throughout this paper, $D_{12}$ represents the mutual diffusion coefficient and an italicized $D_{12}$ represents the kinetic part sans $Q$. While intradiffusion coefficients accurate to approximately $3 \%$ are often obtained from MD simulations, comparable statistics for mutual diffusion coefficients would require runs longer by at least a factor of $N$, perhaps considerably longer due to the necessity of separating time averages in phase space to ensure their independence. ${ }^{3}$

Using the Green-Kubo relationship [Eq. (3)], Jacucci and McDonald ${ }^{1}$ (JM) obtained mutual diffusion coefficients for an equimolar mixture of argon and krypton using the Lennard-Jones ( $\mathrm{LJ}$ ) pair potential and Lorentz-Berthelot (LB) combining rules:

$$
\sigma_{12}=\frac{1}{2}\left(\sigma_{1}+\sigma_{2}\right)
$$

and

$$
\epsilon_{12}=\left(\epsilon_{1} \epsilon_{2}\right)^{1 / 2} \text {. }
$$

The emphasis in the JM study was on self-diffusion coeffcients and no attempt was made to improve the statistics of the mutual diffusion coefficients. If Eq. (4) is restructured, the mutual diffusion coefficient of a binary mixture can be written as ${ }^{3}$ 


$$
D_{12} / Q=x_{2} D_{1}+x_{1} D_{2}+k T\left(\frac{x_{2}}{m_{1}}+\frac{x_{1}}{m_{2}}\right) \int_{0}^{\infty} \Upsilon(t) d t
$$

to explicitly show its relationship to tracer diffusion coefficients (involving only self-correlation integrals) and a deviation term involving $\Upsilon(t)$, a sum of all cross correlations of the type $\left\langle\mathbf{v}_{i}(0) \cdot \mathbf{v}_{j}(t)\right\rangle$. The magnitude of this cross correlation term was smaller than the statistical uncertainties in the JM simulations.

Jolly and Bearman ${ }^{2}$ (JB) using the MSD method also computed mutual diffusion coefficients of the same system by averaging the results of numerous 7500 -time-step runs. They report an uncertainty of about $8 \%$ in their results. However, by analyzing methods for decreasing the uncertainty in calculated mutual diffusion coefficients, Schoen and Hoheisel ${ }^{3}$ (SH) found that the MSD runs of JB did not contain sufficient independent time origins to calculate accurate time correlation functions. Time origins are independent when separated in time by the decay length of the velocity correlation function, about 20 time steps in the runs by SH. The number of independent time origins is more important in reducing the uncertainty of $D_{12}$ than the number of averages because averaging shorter runs retains systematic errors inherent in shorter runs. The results of $\mathrm{SH}$ indicate that approximately 2000 independent time origins (generally about 50000 time steps) are required to obtain $D_{12}$ values accurate to $3 \%-4 \%$ compared to the roughly 350 used in the JB study. The SH results clearly indicate that accurate mutual diffusion coefficients can be calculated from sufficiently long simulations.

The magnitude of cross correlations can be particularly emphasized in terms of the ratio $D_{12} / D_{12}^{0}$ where $D_{12}^{0}$ is a "pure mutual diffusion coefficient" 3 defined for a binary mixture as

$$
D_{12}^{0}=Q\left(x_{2} D_{1}+x_{1} D_{2}\right)
$$

Toukubo and Nakanishi ${ }^{7}$ studied the effect of varying $\epsilon_{12}$ on intradiffusion coefficients and found that decreasing $\epsilon_{12}$ increased the intradiffusion coefficient and vice versa. Similarly, Nakanishi et al. ${ }^{8}$ found that mutual diffusion coefficients in simple model mixtures increase with decreasing $\epsilon_{12}$ values. Although the statistical uncertainties in $D_{12} / D_{12}^{0}$ were relatively large (the work predated the SH work), it was apparent from their values that cross interaction terms affect mutual diffusivity more than tracer diffusion coefficients because of their significant effect upon the cross correlation function. These results, combined with the findings by SH that substantial differences between the $\mathrm{LJ}$ parameters of the two pure components (asymmetries) have a significant effect upon the magnitude and sign of the cross correlation functions, imply that $\mathrm{LJ}$ parameters must be selected carefully for accurate simulation of $D_{12}$ in real mixtures of more complex molecules in which significant departures from Eq. (10) are often observed.

In this work, mutual diffusion coefficients in mixtures of $n$-hexane, $n$-heptane, $n$-octane, and 3 -methylpentane with carbon tetrachloride are simulated with $\mathrm{MD}$ as a function of composition, and the results are compared to experimental data. $^{9}$ To our knowledge, mutual diffusion coefficients of such systems have not been studied by molecular dynamics. The purpose of the simulations is to test the feasibility of using MD simulations of molecules interacting with $\mathrm{LJ}$ potentials to predict experimental mutual diffusion coefficients of real systems. As a matter of course, related questions must also be addressed by this study: (i) are effective LJ parameters adequate to simulate diffusion coefficients in mixtures containing nonspherical molecules, (ii) are LB combining rules adequate to explain the observed composition dependence of mutual diffusion coefficients in real mixtures, and (iii) what is the effect of $\sigma_{12}$ cross interactions on the mutual diffusion coefficient?

\section{SIMULATION CONDITIONS}

Mutual diffusion coefficients in this work were obtained from the GK formalism using Eq. (3). Each simulation was made on a system of 216 particles using NVE MD with a potential cutoff distance of $2.5 \sigma$. Standard techniques for periodic boundary conditions, neighborhood lists, and longrange corrections were also used. Initial configurations were generated by placing molecules, randomly selected in proportion to the desired mole fraction, on lattice sites. Initial velocities were generated randomly from a Gaussian distribution. Subsequently, a 2000 time-step cycle containing equilibration steps followed by a temperature rescale was repeated until compositional and thermal equilibria were reached at the desired starting conditions. Then an additional 5000 time-step equilibration segment was run before starting the velocity correlation computations. The Verlet Leapfrog algorithm was used to numerically integrate the equations of motion with a time step of $0.01 \mathrm{ps}$. The total length of the simulations was between 90000 and 120000 time steps. Records of the velocity correlation functions were taken during periods of 10000 time steps with a 5000 time step separation between periods. The separation sequence was probably not necessary but was included to extend sampling over a larger region of phase space. Each run therefore contains between 2400 and 3200 independent time origins, well within the requirements prescribed by $\mathrm{SH}$. Within each 10000 -time-step block, the correlation function accumulations were started every time step and collected for 600 time steps ( 6 ps in real time).

In theory, the value of the diffusion coefficient is determined from the infinite-time GK integral. However, a real simulation must be stopped at some finite time and the integral evaluated based on the length of the simulation. As indicated by Michels and Trappeniers, ${ }^{10}$ the finite upper integration limit must be chosen so that the contribution of the remaining long-time tail is small compared to the desired statistical accuracy of the integral. Longer times are required for fluctuations of the center-of-mass correlation function to dampen than for their single-particle analogues, dictating longer correlation calculations for mutual diffusion coefficients. Values for each simulation were determined from the average of the integral between 3 and 6 ps. This time was found to be adequate for complete decay of the correlation function.

To check the accuracy of the method and code, simulations were made at the conditions of JM, JB, and SH on a 50 
TABLE I. Comparison of simulated diffusion coefficients for an argonkrypton equimolar mixture at $V=33.31 \mathrm{~cm}^{3} / \mathrm{mol}$ and $T^{*}=0.965$.

\begin{tabular}{lcccc}
\hline \hline & \multicolumn{4}{c}{$\left(10^{-5} \mathrm{~cm}^{2} / \mathrm{s}\right)$} \\
\cline { 2 - 5 } & This study & $\mathrm{JM}$ & $\mathrm{JB}$ & $\mathrm{SH}$ \\
\hline$D_{\mathrm{Ar}}$ & 2.97 & 3.01 & 3.16 & 2.97 \\
$D_{\mathrm{Kr}}$ & 2.42 & 2.40 & 2.61 & 2.44 \\
$D_{12}$ & 2.81 & 2.80 & $2.71^{\mathrm{a}}$ & 2.86 \\
\hline
\end{tabular}

a The number reported by JB is 2.63 using a value for $Q$ of 0.97 . All of the other values in the table use a value of 1.0 for $Q$ so the $\mathrm{JB}$ value was adjusted to the same basis. Additionally, SH cite in a footnote a private communication from Professor Bearman. Professor Bearman discovered a small coding error in the program used to obtain the $\mathrm{JB}$ results. Correction of this error brings the JB and SH results into "good agreement" according to the footnote.

mol \% mixture of $\mathrm{Ar}$ and $\mathrm{Kr}$. In order to make a direct comparison, a small correction for the number dependence was included in the calculations by using the values obtained in the JB study for systems ranging in size from 108 to 864 particles. A direct comparison of the results given in Table I shows excellent agreement.

The values of the molecular weights and densities used for the simulations in this work are given in Table II. All of the simulations were made at $30^{\circ} \mathrm{C}$ to correspond to the temperature of the experimental results. Most of the simulations were run on a convex $\mathrm{C} 210$ or a Harris 8600 machine. One time step took about $0.96 \mathrm{~s}$ of CPU time on the Convex and about $5.0 \mathrm{~s}$ on the Harris machine. Simulation lengths of at least 60000 time steps greatly attenuated the fluctuations in the correlation functions in agreement with the findings of SH. The reported values are expected to have a standard

TABLE II. Densities and thermodynamic factors of alkanes in carbon tetrachloride at $30^{\circ} \mathrm{C}$

\begin{tabular}{llll}
\hline \hline Component & $x_{1}$ & $\rho_{m}\left(\mathrm{~g} / \mathrm{cm}^{3}\right)$ & $Q$ \\
\hline n-Hexane & 0.0 & 1.5635 & 1.00 \\
& 0.1 & 1.4447 & 0.96 \\
& 0.2 & 1.3336 & 0.94 \\
& 0.5 & 1.0397 & 0.94 \\
& 0.8 & 0.7936 & 0.98 \\
n-Heptane & 0.9 & 0.7202 & 1.00 \\
& 1.0 & 0.6506 & 1.00 \\
& 0.0 & 1.5635 & 1.00 \\
& 0.1 & 1.4354 & 0.95 \\
& 0.5 & 1.0276 & 0.95 \\
n-Octane & 0.9 & 0.7342 & 0.99 \\
& 1.0 & 0.6733 & 1.00 \\
& 0.0 & 1.5635 & 1.00 \\
& 0.1 & 1.4266 & 0.98 \\
& 0.2 & 1.3060 & 0.97 \\
& 0.5 & 1.0174 & 0.96 \\
& 0.8 & 0.8050 & 0.97 \\
& 0.9 & 0.7461 & 0.98 \\
& 1.0 & 0.6920 & 1.00 \\
& 0.0 & 1.5635 & 1.00 \\
& 0.1 & 1.4451 & 0.96 \\
& 0.5 & 1.0409 & 0.94 \\
& 0.9 & 0.7215 & 1.00 \\
& 1.0 & 0.6519 & 1.00 \\
\hline \hline
\end{tabular}

error of about $5 \%-6 \%$ based on the standard deviation of averages calculated separately from three subsections of each long run.

\section{PURE-COMPONENT LJ PARAMETERS}

It is well known that several equally valid sets of $\sigma$ and $\epsilon$ parameters may result when the parameters are regressed from experimental data. Generally, any one set is adequate for calculating properties similar to those from which the parameters were regressed. However, much poorer results are obtained when parameters regressed from one property are used to predict another. Two problems are apparent: (i) nonuniqueness of the parameters and (ii) nonuniversality of the parameters for different properties. The first problem arises from coupling between the $\sigma-\epsilon$ parameters so that multiple sets may fit the data equally well. ${ }^{11,12}$ This coupling generally implies that $\epsilon$ must increase as $\sigma$ is decreased, and vice versa. ${ }^{13} \mathrm{Hu}$ et al. ${ }^{14}$ attempted to decouple $\sigma$ and $\epsilon$ to obtain unique values by simultaneously regressing viscosity, second virial coefficient and self-diffusion data. They did not find a unique solution, but they were able to obtain a satisfactory set of parameters which slightly compromised the accuracy of all three properties to minimize the total error. This typifies the nonuniversality problem, a problem presumed to be due to modeling nonspherical molecules with spherical potentials. By doing so, only effective $\mathrm{LJ}$ parameters are obtained. Because structure and intermolecular forces affect properties differently, effective spherical parameters will not be universal.

Convincing evidence of the inadequacy of using virial coefficient data to compute $D_{12}$ is shown in Fig. 1 where MSD results from a simulation of $n$-hexane in carbon tetrachloride at $30^{\circ} \mathrm{C}$ and $1 \mathrm{~atm}$, using $\mathrm{LJ}$ parameters obtained from second virial coefficient data, flatten after about $0.8 \mathrm{ps}$. This drop in the resultant diffusion coefficient is indicative of freezing as the molecules become caged at their lattice sites. These LJ parameters are obviously inconsistent with the phase behavior of the system at $30^{\circ} \mathrm{C}$.

Assuming the lack of universality is in fact due to the different influence of effective potentials on different properties, LJ parameters determined from diffusive phenomena

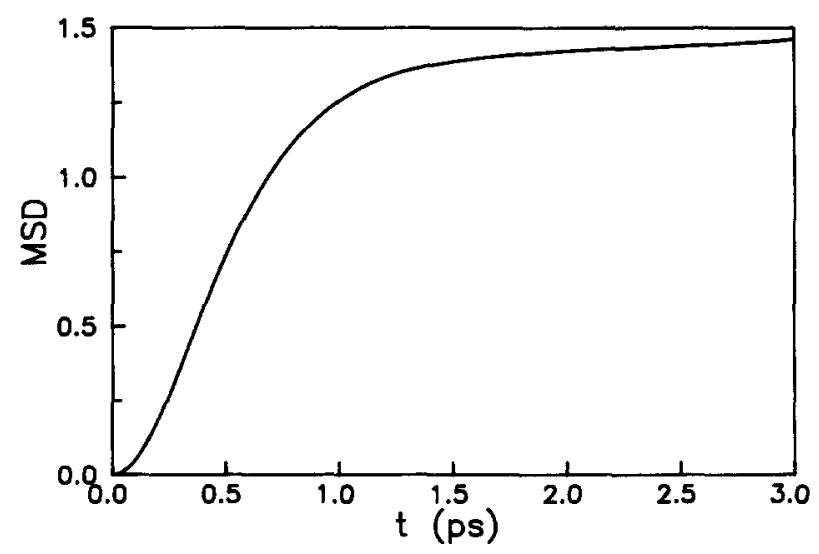

FIG. 1. MSD as a function of time for a $50 \mathrm{~mol} \%$ mixture of $n$-hexane in carbon tetrachloride at $30^{\circ} \mathrm{C}$ using $\mathrm{LJ}$ parameters obtained from virial coefficient data. The change in slope reflects freezing. 


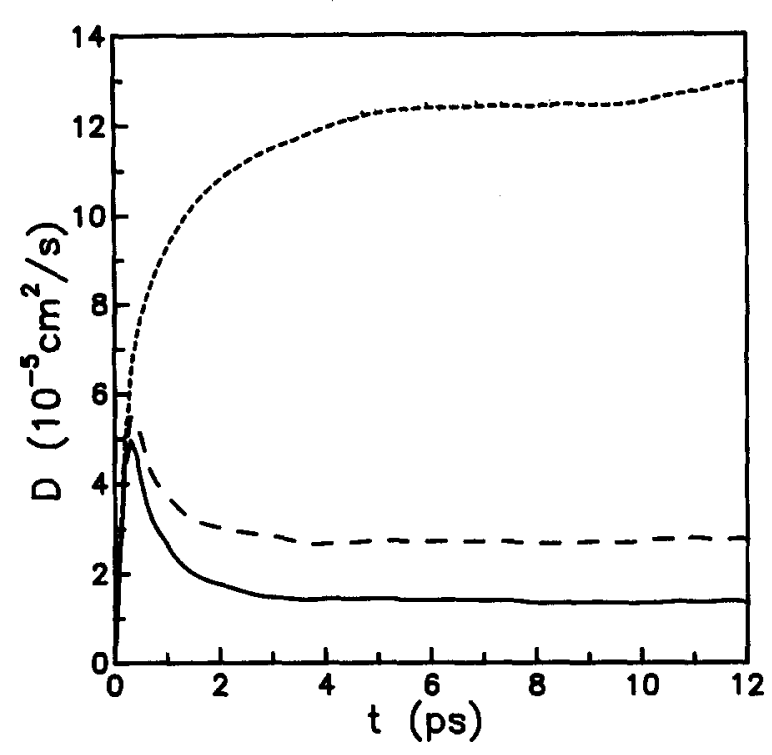

FIG. 2. Sensitivity of the integral of the velocity autocorrelation function for pure $n$-hexane to changes in the $\mathrm{LJ}$ parameters: $(-)$, both parameters obtained from viscosity data; $(--), \epsilon$ decreased by $25 \%$; and $(---), \sigma$ decreased by $25 \%$.

(mutual and tracer diffusion) should be consistent. For this reason self-diffusion coefficients (tracer diffusion coefficients at the pure component limit) were used in this work to obtain pure component $\mathbf{L J}$ parameters. The uniqueness problem was avoided by using the value of $\epsilon$ obtained from viscosity $(\eta)$ data and adjusting $\sigma$. The reported relative constancy ${ }^{15}$ of $\eta D$ indicates some congruity between the influences of effective $L J$ parameters on $\eta$ and $D$. This was also borne out by the multiproperty study of $\mathrm{Hu}$ et al. Simulations were made to determine the relative sensitivities of selfdiffusion coefficients to variations in $\epsilon$ and $\sigma$. The values of $\sigma$ and $\epsilon$ were independently varied from the viscosity values reported in the literature for pure $n$-hexane. Each parameter was independently reduced by about $25 \%$ and the self-diffusion coefficients were calculated from MD simulations. The results, shown in Fig. 2, indicate that the diffusion coefficient is much more sensitive to variations in $\sigma$ than in $\epsilon$. Furthermore, it is evident that only $\sigma$ or $\epsilon$ need be adjusted, not both, to obtain appropriate values of the self-diffusion coefficient. Thus, pure component LJ parameters were obtained by using the viscosity-regressed values of $\epsilon$ and adjusting $\sigma$ until simulated and experimental values agreed. By changing only the most sensitive parameter, $\sigma$, effective parameters were obtained which are as close as possible to those regressed from viscosity data. When no $L J$ parameters were available in the literature, $\epsilon$ values were calculated using the correlation of Chung ${ }^{16}$ :

$$
\frac{\epsilon}{k}=\frac{T_{c}}{1.2593} \text {. }
$$

The self-diffusion coefficient values used in the regresion, the values of $\epsilon$ obtained from the literature, and the resultant values obtained for $\sigma$ are given in Table III. Selfdiffusion coefficients were calculated for different $\sigma$ values until the experimental $D_{i}$ value was bracketed. The optimum
TABLE III. Values for $\sigma$ obtained from experimental self-diffusion coeffcients at $30^{\circ} \mathrm{C}$ using $\epsilon / k$ values from viscosity data.

\begin{tabular}{lccc}
\hline \hline Component & $D_{i}\left(10^{-5} \mathrm{~cm}^{2} / \mathrm{s}\right)$ & $\sigma(\AA)$ & $\epsilon / k(\mathrm{~K})^{\mathrm{a}}$ \\
\hline Carbon tetrachloride & $1.43^{\mathrm{b}}$ & 5.378 & 327.0 \\
$n$-Hexane & $4.37^{\mathrm{c}}$ & 5.645 & 413.0 \\
$n$-Heptane & $3.20^{\mathrm{d}}$ & 5.955 & 429.0 \\
$n$-Octane & $2.41^{\mathrm{d}}$ & 6.323 & 320.0 \\
3-Methylpentane & $3.76^{\mathrm{d}}$ & 5.700 & 400.5 \\
\hline
\end{tabular}

Reference 15.

${ }^{b}$ Reference 17.

' Reference 18.

${ }^{\mathrm{d}}$ Reference 19.

value of $\sigma$ was then obtained from a quadratic fit of the data bracketing the experimental value. Each point was obtained by averaging four to six replicates from simulations of at least 10000 time steps at a temperature of $30^{\circ} \mathrm{C}$. The additional computational asset of using fewer particles than in previous studies is gained by using potential parameters obtained from self-diffusion coefficients. Both SH and JB have shown that any dependence of the results on particle number is the same for both intra- and mutual diffusion coefficients.

\section{RESULTS AND DISCUSSION}

A direct comparison of $\mathrm{MD}$ results to experimental data requires values of the thermodynamic factor $Q$ shown in Eq. (6). In previous studies ${ }^{2,3} \mathrm{MD}$ was used to calculate not only the GK time correlation functions but also $Q$. Unfortunate$1 y$, it is difficult to obtain good statistics in MD determinations of the chemical potential itself ${ }^{4}$ and $Q$ depends on the derivative of chemical potential with respect to composition. The uncertainty in $Q$ obtained in the previous studies was greater than the deviations from Eq. (10). In this work values of $Q$ were calculated from values of $\ln \gamma_{1}$ obtained from

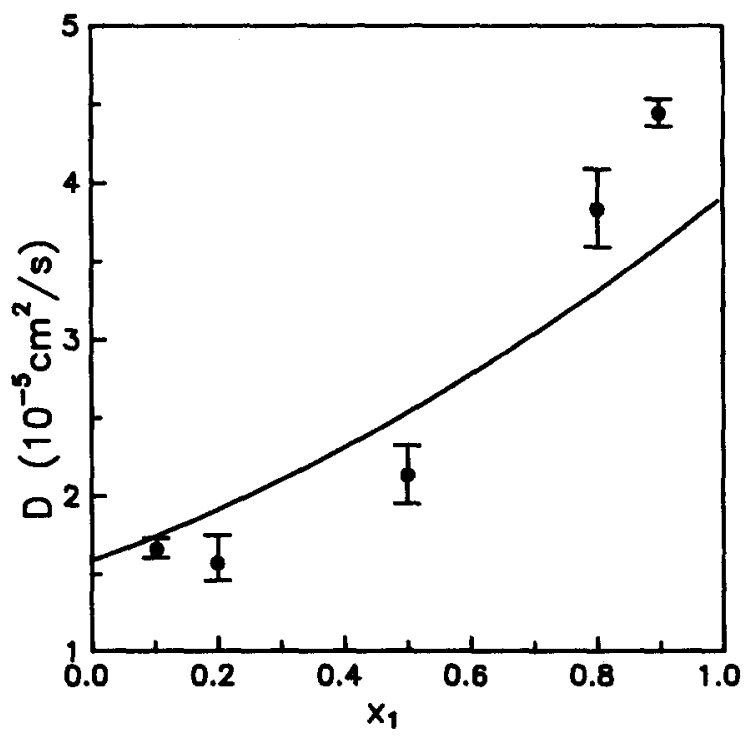

FIG. 3. Comparison of diffusion coefficients simulated using the LB combining rules (points with estimated error bars) for mixtures of $n$-hexane and carbon tetrachloride at $30^{\circ} \mathrm{C}$ to smoothed experimental (line) data. 
TABLE IV. Comparison of predicted binary diffusion coefficients with experimental results for selected concentrations of alkanes in carbon tetrachloride at $30^{\circ} \mathrm{C}$.

\begin{tabular}{|c|c|c|c|c|}
\hline \multirow[b]{2}{*}{ (Comp. 1) } & \multirow[b]{2}{*}{$x_{1}$} & \multicolumn{2}{|c|}{$\left(10^{-5} \mathrm{~cm}^{2} / \mathrm{s}\right)$} & \multirow[b]{2}{*}{ \%Error } \\
\hline & & $D_{12}(\mathrm{MD})$ & $D_{12}$ (Expt.) $)^{\mathrm{a}}$ & \\
\hline$n$-Hexane & $\begin{array}{l}0.1 \\
0.2 \\
0.5 \\
0.8 \\
0.9\end{array}$ & $\begin{array}{l}1.63 \\
1.86 \\
2.57 \\
3.32 \\
3.69\end{array}$ & $\begin{array}{l}1.74 \\
1.91 \\
2.53 \\
3.37 \\
3.60\end{array}$ & $\begin{array}{r}-6.32 \\
-2.62 \\
1.58 \\
-1.48 \\
2.50\end{array}$ \\
\hline$n$-Heptane & $\begin{array}{l}0.1 \\
0.5 \\
0.9\end{array}$ & $\begin{array}{l}1.39 \\
2.16 \\
3.18\end{array}$ & $\begin{array}{l}1.64 \\
2.27 \\
3.00\end{array}$ & $\begin{array}{r}-15.24 \\
4.85 \\
6.00\end{array}$ \\
\hline n-Octane & $\begin{array}{l}0.1 \\
0.5 \\
0.9\end{array}$ & $\begin{array}{l}1.32 \\
2.05 \\
2.49\end{array}$ & $\begin{array}{l}1.47 \\
2.03 \\
2.53\end{array}$ & $\begin{array}{r}-10.20 \\
0.98 \\
-1.58\end{array}$ \\
\hline 3-Methylpentane & $\begin{array}{l}0.1 \\
0.5 \\
0.9\end{array}$ & $\begin{array}{l}1.40 \\
2.38 \\
3.55\end{array}$ & $\begin{array}{l}1.64 \\
2.47 \\
3.66\end{array}$ & $\begin{array}{r}-14.63 \\
-3.64 \\
-3.01\end{array}$ \\
\hline \multicolumn{4}{|c|}{$\begin{array}{r}\text { average absolute error }= \\
\text { bias }=\end{array}$} & $\begin{array}{r}5.33 \% \\
-3.75 \%\end{array}$ \\
\hline
\end{tabular}

${ }^{\mathrm{a}}$ Reference 9.

UNIFAC. ${ }^{15}$ The systems studied are fairly ideal and values of $Q$ are all reasonably close to 1.0 as shown in Table II.

The binary systems $n$-hexane, $n$-heptane, $n$-octane, and 3-methylpentane in carbon tetrachloride at $30^{\circ} \mathrm{C}$ were studied as a function of composition. This sequence of alkane solutes was chosen to examine the efficacy of using MD and the $\mathrm{LJ}$ potential to predict not only the compositional dependence of $D_{12}$ but also its dependence on alkane chain length and branching. Values of the mutual diffusion coefficients obtained for the $n$-hexane + carbon tetrachloride system using LB combining rules are compared to smoothed experimental data ${ }^{9}$ in Fig. 3. The average absolute error for all of the points is $14.7 \%$ with a bias of $+4.8 \%$. There is obviously a systematic error resulting in larger deviations at higher

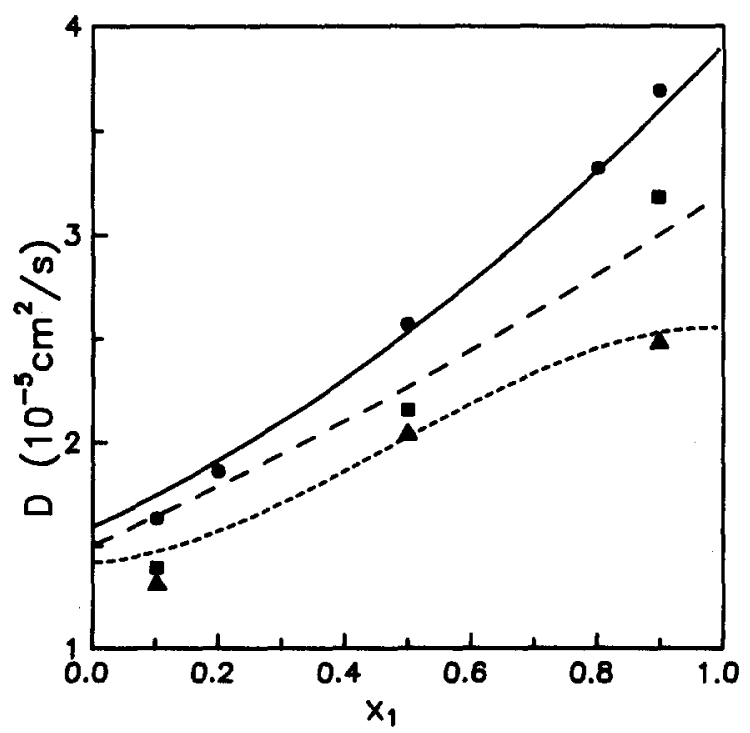

FIG. 4. Mutual diffusion coefficients at $30^{\circ} \mathrm{C}$ for $n$-hexane $(O,-)$, n-heptane $(\boldsymbol{n},--)$, and $n$-octane $(\mathbf{\Lambda},--)$ ) in carbon tetrachloride. Points are simulated values and lines are smoothed experimental data. alkane mole fractions. The intradiffusion coefficients do approach the self-diffusion coefficient values in the pure component limit. This is expected since the $\mathbf{L J}$ parameters were determined from self-diffusion coefficient data. However, at the opposite composition limit where $D_{12}$ should approach the tracer diffusion coefficient, there is significant inconsistency between the calculated intradiffusion values and the experimental infinite dilution mutual diffusion coefficients. One likely cause of this disparity is use of the empirical LB combining rule. As has been pointed out, both the tracer coefficients and the cross correlation term are sensitive to the choice of the cross interactions, particularly $\sigma_{12}$.

One possiblity of obtaining agreement with experiment is to abandon the empirical LB combining rule and adjust the cross interactions. A systematic study of varying $\epsilon_{12}$ and $\sigma_{12}$ was not made. Rather, only $\sigma_{12}$ was adjusted because of the much larger sensitivity of the diffusion coefficient to it than $\epsilon_{12}$. The value of $\epsilon_{12}$ was still obtained from Eq. (8). Our purpose here is only to show that adjusting $\sigma_{12}$ can bring experiment and theory into agreement over the entire composition range; we have not attempted to demonstrate that this is the only remedy. Focusing only on the 0.9 mole fraction point of the hexane-carbon tetrachloride system, the combining rule

$$
\sigma_{12}=\sigma_{1}+0.75\left(\sigma_{2}-\sigma_{1}\right)
$$

was found to be adequate. Here subscript 1 refers to carbon tetrachloride and 2 refers to $n$-hexane. This combining rule, estimated from the diffusion coefficient at a single composition, was then used to calculate cross interaction parameters for the remaining compositions and systems. Table IV compares the simulated values to smoothed experimental data. The average absolute error for the fourteen simulated points is $5.3 \%$ with a bias of $-3.8 \%$. Most of this negative bias is directly attributable to the $10 \mathrm{~mol} \% \mathrm{CCl}_{4}$ points for which a small systematic deviation is still evident in Fig. 4.

As Fig. 4 shows, the agreement of the simulated results with experimental data is quite good over the whole composition range except at the very low alkane concentrations. It should be clarified here that these simulations are predictions not correlations. It can be seen from Eq. (10) that the mutual diffusion coefficient approaches the infinite dilution tracer diffusion coefficient in the pure component limitthese are not the self-diffusion coefficient values which are defined at the opposite concentration limit. There is no known rigorous relation between self and mutual diffusion coefficients. The pure component $\mathrm{LJ}$ parameters were obtained from self-diffusion coefficients, not infinite dilution tracer diffusivities, and therefore this procedure does not empirically adjust parameters using experimental values of $D_{12}$.

The decrease in the mutual diffusion coefficient with increasing alkane chain length, possibly predominated by molecular weight rather than structural effects, is accurately predicted by the MD simulations as shown in Fig. 4. The effect of chain branching is a much smaller effect and is shown in Fig. 5. The qualitative features of the influence of chain branching are again reproduced by the MD simulation, although the fine differences in the composition depen- 


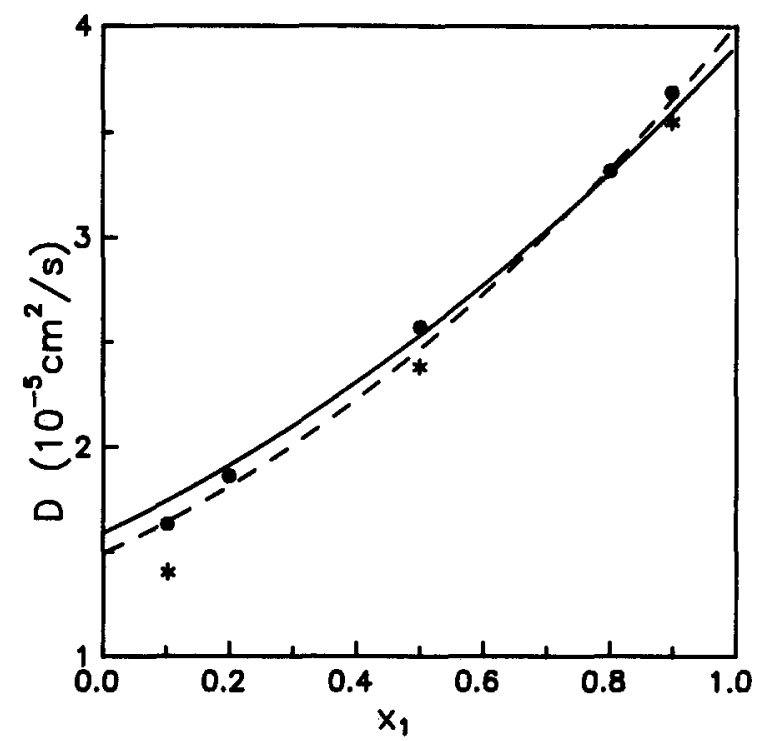

FIG. 5. Mutual diffusion coefficients at $30^{\circ} \mathrm{C}$ for $n$-hexane $(\bullet,-)$ and 3methylpentane $(*,-)$ in carbon tetrachloride. Points are simulated values and lines are smoothed experimental data.

dence of $D_{12}$ for the two systems is not within the resolution capability of these MD simulations.

The combining rule used for $\sigma_{12}$ for these alkane mixtures weights the alkane contribution more heavily. The required weighting is probably due to the larger effective spherical volume swept out by the rotating cigar-shaped alkane molecules as they interact with the more spherical carbon tetrachloride molecules. It is interesting that weighting the combining rule toward the alkane component improved the simulated results in the alkane-rich compositions without significantly altering the results in the carbon tetrachloride-rich region. The larger effect of the weighted combining rule upon $D_{12}$ in the alkane-rich region can also be seen in the center of mass velocity correlation functions shown in Fig. 6 for $n$-octane mixtures with carbon tetrachloride. As one would expect, the negative backscatter peak decreases (the diffusion coefficient increases) with decreasing density (higher alkane compositions). This is because at the higher alkane concentrations, the lower density permits more freedom for molecular motion and the effect of the liquid cage structure is diminished. Upon close examination of Fig. 6, it can be seen that the effects of the change in the mixing rule are more dominant in the alkane-rich region. It also appears that in the mid- and high-density regions, the degree of backscatter is less for the weighted combining rule than for LB. This has the net effect of decreasing $D_{12}$ in the alkane-rich region and increasing it in the middle region relative to the $L B$ rule, clearly the effect desired upon consideration of Fig. 2.

Table $\mathrm{V}$ contains values of the intradiffusion coefficients obtained from the simulations. The uncertainty of these coefficients is about $2 \%$. Also shown in this table are the kinetic diffusion coefficients (sans $Q$ ) $D_{12}, D_{12}^{0}$ and the ratio $D_{12} / D_{12}^{0}$. As stated earlier, the cross correlation effects are relatively large in real mixtures of complex molecules, reach-

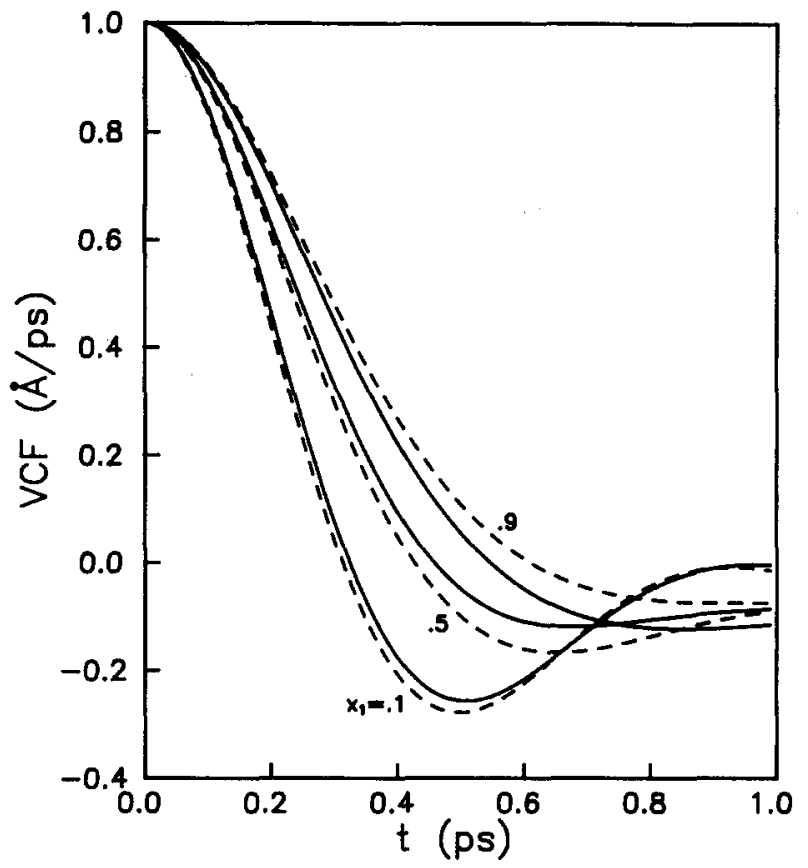

FIG. 6. Center of mass velocity correlation function for $n$-octane in carbon tetrachloride at $30^{\circ} \mathrm{C}$ using the weighted (-) and the $\mathrm{LB}(--)$ combining rules for $\sigma_{12}$.

ing nearly $60 \%$ in the case of $n$-octane and carbon tetrachloride. The cross correlation effect, qualitatively similar in all four systems, is highest at lower alkane compositions, remains fairly high in the mid-composition regions, and then drops more rapidly toward unity at higher alkane compositions. The magnitude of the cross correlation is much smaller for the more branched alkane. Values of $D_{12} / D_{12}^{0}$ are considerably smaller for the LB combining rule supporting the conclusions of Nakanishi et al. and SH concerning the sensitivity of the cross correlations to the cross interactions and the asymmetries in pure component properties.

TABLE V. Kinetic intradiffusion, pure mutual diffusion, mutual diffusion and cross correlation coefficients from MD simulations of alkanes in carbon tetrachloride at $30^{\circ} \mathrm{C}$

\begin{tabular}{lcccccc}
\hline \hline & & \multicolumn{5}{c}{$10^{-5} \mathrm{~cm}^{2} / \mathrm{s}$} \\
\cline { 3 - 6 } Component 1 & $x_{1}$ & $D_{1}$ & $D_{2}$ & $D_{12}^{0}$ & $D_{12}$ & $D_{12} / D_{12}^{0}$ \\
\hline n-Hexane & 0.1 & 1.39 & 1.52 & 1.40 & 1.70 & 1.21 \\
& 0.2 & 1.58 & 1.81 & 1.63 & 1.98 & 1.21 \\
& 0.5 & 2.28 & 2.51 & 2.40 & 2.73 & 1.13 \\
& 0.8 & 3.30 & 3.40 & 3.38 & 3.39 & 1.00 \\
& 0.9 & 3.89 & 3.85 & 3.85 & 3.69 & 0.96 \\
n-Heptane & 0.1 & 1.15 & 1.40 & 1.18 & 1.46 & 1.24 \\
& 0.5 & 1.55 & 1.90 & 1.73 & 2.27 & 1.31 \\
& 0.9 & 2.69 & 2.97 & 2.94 & 3.21 & 1.09 \\
n-Octane & 0.1 & 0.81 & 1.27 & 0.86 & 1.35 & 1.57 \\
& 0.5 & 1.26 & 1.55 & 1.41 & 2.13 & 1.51 \\
& 0.9 & 2.11 & 2.38 & 2.35 & 2.54 & 1.08 \\
3-Methylpentane & 0.1 & 1.27 & 1.44 & 1.29 & 1.46 & 1.13 \\
& 0.5 & 2.10 & 2.30 & 2.20 & 2.53 & 1.15 \\
& 0.9 & 3.37 & 3.42 & 3.42 & 3.55 & 1.04 \\
\hline \hline
\end{tabular}




\section{CONCLUSIONS}

We conclude from this study that effective $\mathrm{LJ}$ potential parameters can be used to predict experimental mutual diffusion coefficients if the pure component parameters are obtained from closely related diffusion processes. Reasonably accurate mutual diffusion coefficients were predicted over the entire composition range from pure component self diffusion data for the systems studied. However, an adequate mixing rule is also required for the cross interaction terms. The LB combining rule was inadequate for the alkane + carbon tetrachloride system. While no attempt was made to regress $\sigma_{12}$ and $\epsilon_{12}$, the higher sensitivity of $D_{12}$ to $\sigma$ than $\epsilon$ inferred that a weighted average of pure $\sigma$ with the standard LB combining rule for $\epsilon$ might be sufficient to produce agreement with experiment. The chosen weighted combining rule was effective for all of the mixtures studied here. Further studies would be useful to more systematically study the effects of the cross interactions on mutual diffusion coefficients.

We take the liberty here of suggesting an extrapolation of our findings to an area of considerable importance. Mutual diffusion coefficients in ternary systems are difficult to measure, particularly the cross terms. Experimental mutual diffusivities in systems of more than three components are virtually nonexistent because of the problems inherent in decoupling the effects of the rapidly increasing (with component number) number of independent diffusion coefficients. It seems that extension of the method utilized here of calculating mutual diffusion coefficients from MD simulations using $L J$ parameters derived from self diffusion coeffi- cients could provide sorely needed multicomponent diffusion information.

\section{ACKNOWLEDGMENT}

Partial support of this project under National Science Foundation Grant No. CBT-85-1424 is gratefully acknowledged.

'G. Jacucci and I. R. McDonald, Physica A 80, 607 (1975).

${ }^{2}$ K. L. Jolly and R. J. Bearman, Mol. Phys. 41, 137 (1980).

${ }^{3}$ M. Schoen and C. Hoheisel, Mol. Phys. 52, 33 (1984); 52, 1029 (1984).

${ }^{4}$ M. P. Allen and D. J. Tildesley, Computer Simulation of Liquids (Clarendon, Oxford, 1987).

${ }^{5}$ W. A. Steele, in Transport Phenomena in Fluids, edited by H. J. M. Hanley (Dekker, New York, 1969), Chap. 8.

6J. P. Hansen and I. R. McDonald, Theory of Simple Liquids (Academic, New York, 1976).

${ }^{7}$ K. Toukubo and K. Nakanishi, J. Chem. Phys. 72, 3089 (1980).

${ }^{9}$ R. L. Rowley, S. C. Yi, D. V. Gubler, and J. M. Stoker, Fluid Phase Equilib. 36, 219 (1987)

${ }^{10}$ J. P. J. Michels and N. J. Trappeniers, Physica A 90, 179 (1978),

"D. Reichenberg, AIChE. J. 19, 854(1973).

${ }^{12}$ R. C. Reid, Chem. Eng. Prog. Monog. Ser. 64, 14 (1968).

${ }^{13}$ L. S. Tee, S. Gotoh, and W. E. Stewart, Ind. Eng. Chem. Fund. 5, 356 (1966).

${ }^{14}$ A. T. Hu, P. S. Chappelear, and R. Kobayashi, AIChE. J. 16, 490 (1970).

${ }^{15}$ R. C. Reid, J. M. Prausnitz, and B. E. Poling, The Properties of Gases and Liquids (McGraw-Hill, New York, 1988).

${ }^{16}$ T.-H. Chung, L. L. Lee, and K. F. Starling, Ind. Eng. Chem. Fund. 23, 8 (1984).

${ }^{17}$ M. A. McCool and L. A. Woolf, J. Chem. Soc. 68, 1971 (1972).

${ }^{18}$ K. R. Harris, J. Chem. Soc. 78, 2265 (1982).

${ }^{19}$ M. R. Riazi and T. E. Daubert, AIChE. J. 26, 386 (1980). 